\title{
Atividades que podem propiciar o desenvolvimento do raciocínio funcional no alunado do ensino médio e universitário inicial
}

\author{
Gilda Palis
}

\section{Resumo}

O conceito de função é certamente uma noção fundamental dentre as estudadas nos cursos de matemática no ensino médio e no início do ciclo universitário na área técnico-científica. Ao mesmo tempo, o ensino e a aprendizagem desse conceito têm sido considerados bastante problemáticos. Neste artigo, discutimos algumas das dificuldades dos alunos desses segmentos escolares com o conceito de função, dentre elas a extrema aderência à concepção de função como expressão algébrica e dificuldades encontradas na resolução de problemas de otimização de enunciado verbal. Sugerimos atividades que podem ser trabalhadas com esse alunado, abrangendo a identificação de funções subjacentes a gráficos estatísticos e problemas de otimização de enunciado verbal que propiciam oportunidades de interpretações qualitativas de aspectos covariacionais da situação funcional estudada e a utilização de diferentes representações de uma mesma função, inclusive de representações digitais.

Palavras-chave: conceito de função; transição ensino médio-superior; gráficos cartesianos e estatísticos; atividades de otimização; recursos digitais no ensino e aprendizagem de funções.

\begin{abstract}
he concept of function is certainly a fundamental notion among those studied in mathematics courses in high school and the beginning of the university cycle in the technical-scientific area. At the same time, the teaching and learning of this concept has been considered quite problematic. In this article, we discuss some of the difficulties of students in these school segments with the concept of function, among them extreme adherence to the conception of function as a algebraic expression and difficulties encountered in solving optimization problems of verbal enunciation. We suggest activities that can be worked out with these students, including the identification of functions underlying statistical graphs and problems of optimization of verbal enunciation that provide opportunities for qualitative interpretations of covariational aspects of the functional situation studied and the use of different representations of the same function, including digital representations.
\end{abstract}

Keywords: concept of function; transition from high school to undergraduation; Cartesian and statistical graphics; optimization activities; digital resources in teaching and learning functions. 


\section{Introdução}

As dificuldades dos alunos com o conceito de função têm sido largamente mencionadas na literatura especializada. Diversos estudos vêm indicando que a aprendizagem desse conceito desenvolve-se ao longo de vários anos e é mais complexa do que se supõe implicitamente no ensino e na concepção de currículos, [1], [3]. Na transição do ensino médio para o superior, um número expressivo de alunos acredita que todas as funções podem ser definidas por uma fórmula algébrica; uma função é somente pensada em termos de manipulação simbólica e numérica, de forma desconectada do seu aspecto conceitual. Essa concepção de função como fórmula é acompanhada da dificuldade em discernir variável de incógnita, função de equação, e da construção de domínio de uma função como sendo um tipo de exercício no qual se "resolvem" denominadores e argumentos de funções raiz quadrada ou logarítmica. Aspectos procedimentais predominam e o desenvolvimento de aspectos conceituais é bastante limitado, [2].

Diversos trabalhos que investigam o ensino e a aprendizagem de funções têm enfatizado também a necessidade de um desenvolvimento do conceito de função que inclua uma concepção de função como covariação de quantidades e não somente como correspondência. Nesse sentido, o que se propõe é que o aluno desenvolva o raciocínio covariacional, isto é, que lhe permita refletir sobre uma situação na qual há duas quantidades que variam, percebendo como uma delas varia em relação à outra. Para isso é importante que o aluno seja exposto a atividades envolvendo o conceito de função como covariação, [7], [15].

A literatura relacionada a problemas de enunciado verbal (word problems) aponta os diversos obstáculos encontrados pelos alunos nesse tipo de problema, dentre eles, a conversão entre diferentes linguagens, o que envolve designar variáveis e distingui-las de constantes, além de representar as correspondências entre as variáveis envolvidas. Temos observado também os problemas enfrentados por alunos recém-ingressos no ensino superior na área técnico-científica em suas buscas de compreensão de textos matemáticos nos quais se misturam língua materna, termos técnicos matemáticos e simbologia matemática, além das características próprias do discurso matemático. Tais fatores interferem no trabalho com problemas que requerem uma compreensão do conceito de função que inclua a habilidade de conceber, representar e interpretar situações funcionais, um aprendizado essencial para o aluno que finaliza o ensino médio e espera prosseguir em seus estudos nas diversas áreas com base matemática do ensino superior, [5].

No planejamento das atividades que procuramos desenvolver para lidar com essa problemática, levamos em conta também um dos objetivos mais citados nas reformas curriculares e instrucionais propostas nos últimos 20 anos: o desenvolvimento conceitual dos alunos pode ser caracterizado pela presença de uma riqueza de conexões. O desenvolvimento de habilidades de produção e coordenação de diferentes representações de um mesmo objeto ou procedimento matemático é visto como uma maneira de construir redes importantes de associações entre essas representações, favorecendo as construções cognitivas. Compartilhando as hipóteses cognitivas teóricas de diversos autores, consideramos que a apreensão conceitual de um objeto matemático é inseparável da apreensão e produção de suas diversas representações. Ser capaz de se mover por diferentes sistemas de representação é uma condição necessária para reconhecer o objeto matemático em cada uma das suas possíveis representações e também para não confundir o objeto conceitual com suas representações, [4].

Diferentes representações de uma mesma função podem permitir processamentos diversos visando a obtenção de informações sobre o seu comportamento. Assim, a utilização de representações múltiplas na resolução de problemas também pode colaborar para o desenvolvimento de processos metacognitivos de autoavaliação pelos alunos; além de usar as representações disponíveis na busca de significados, o aluno pode também comparar as respostas encontradas.

As atividades que serão sugeridas nesse artigo pressupõem alguns aspectos teóricos ligados à terminologia matemática que serão apresentados resumidamente a seguir.

\section{Definição em matemática}


Segundo [16], ao se compreender ou tentar compreender uma frase, as pessoas usualmente não consultam as definições das palavras que a constituem. Até porque muitas palavras do linguajar cotidiano não têm definição (apesar de serem "definidas" de alguma forma em dicionários). Já em contextos técnicos, a atribuição de significado a diversos termos dá-se por estipulação, como em matemática. Nesses ambientes, as definições têm um papel extremamente importante, tanto na construção da imagem mental de um conceito como na resolução de tarefas cognitivas, sendo preciso levá-las em consideração. Assim, contextos técnicos impõem hábitos de pensamento totalmente distintos daqueles típicos dos contextos do dia a dia. É possível antecipar que hábitos do cotidiano podem sobrepor-se a comportamentos necessários em contextos técnicos, [6].

A maior parte dos alunos de ensino médio e inicial universitário não consulta ou se apoia em definições quando trabalha com tarefas matemáticas que lhes são propostas. Hábitos de pensamento do cotidiano assumem o controle das ações empreendidas, e as definições podem ser ignoradas mesmo quando as questões que lhes são propostas requerem que sejam levadas em conta. Assim, esses alunos não desenvolvem hábitos de pensamento que são essenciais em contextos técnicos nos quais as consultas às definições são esperadas. Além disso, o desconhecimento da terminologia dificulta a comunicação entre alunos e professores em sala de aula.

O desenho das atividades que serão expostas nesse artigo pressupõe que os alunos já foram introduzidos às definições de função real de variável real, de gráfico de tal função em um sistema de coordenadas cartesianas ortogonais e de aproximação numérica. De fato, supomos que os alunos têm conhecimento de alguma variante das definições apresentadas abaixo. Consideramos que é importante trabalhar com o suporte dessas definições, reconhecendo as suas componentes, apesar de sabermos que a boa apreensão conceitual ultrapassa em muito esse conhecimento.

Uma função real $f$ definida em um subconjunto $D$ dos números reais, denotada por $f: D \subset \mathbb{R} \rightarrow \mathbb{R}$, é uma correspondência que, a cada número $x$ pertencente a $D$, associa um único número $f(x)$. O conjunto $D$ é chamado domínio da função $f$.

O gráfico de $f: D \subset \mathbb{R} \rightarrow \mathbb{R}$ é o conjunto de pares ordenados $\{(x, f(x)), x \in D\}$. Fixado um sistema de coordenadas cartesianas, um esboço geométrico do gráfico de $f$ é um desenho desse conjunto de pontos. Para não sobrecarregar o texto, a expressão "um gráfico de $f$ " ${ }^{1}$ será empregada com o significado de "um esboço geométrico do gráfico de $f$ " e não de "o conjunto de pares $\{(x, f(x)), x \in D\}$. De fato, há uma infinidade de gráficos de uma mesma função, pois esses dependem de restrições em seus domínios, escolhas de escalas nos eixos coordenados, aproximações numéricas intrínsecas ao contexto gráfico etc., tanto com papel e lápis como usando tecnologia digital. Um desenvolvimento dessas ideias pode ser encontrado em [9] e [10].

Com relação ao conceito de aproximação numérica, podemos raciocinar com a definição abaixo. Maiores detalhes e exemplos podem ser encontrados em [8].

"Dizemos que um número real $a$ é uma aproximação do número real $b$, com erro menor do que $E$, se $|a-b|<E$, ou seja, se, na reta numérica, a distância entre $a$ e $b$ for menor do que $E$."

É importante observar também que, se o domínio de uma função não é fornecido juntamente com a sua relação de correspondência (expressão algébrica ou verbal), adotamos a convenção usual. Assumimos que o seu domínio é o conjunto dos números reais para os quais a correspondência faz sentido.

A seguir, vamos exemplificar atividades instrucionais que podem ser propostas a alunos no segmento de ensino aqui considerado e que tem por objetivo lidar com alguns dos problemas mencionados. Na Seção Gráficos Estatísticos e Funções, as sugestões apresentadas têm por objetivo modificar a restrição conceitual "função é fórmula", chamando a atenção para a riqueza de exemplos de situações funcionais

\footnotetext{
${ }^{1}$ Estaremos nos referindo a esses gráficos pela expressão "gráficos cartesianos", para diferenciá-los de gráficos estatísticos.
} 
subjacentes a gráficos estatísticos que não podem ser expressas por fórmulas algébricas. A Seção Um Problema de Otimização também trabalha inicialmente com uma situação funcional que não se apresenta expressa por uma fórmula. A atividade procura promover o desenvolvimento mais amplo da concepção de função, abrangendo interpretações qualitativas de aspectos covariacionais da situação funcional estudada, a utilização de diferentes representações de uma mesma função, inclusive de representações digitais. Para alguns aspectos teóricos que embasam esse desenvolvimento, ver [11] e [12].

\section{Gráficos estatísticos e funções}

Funções são criadas para estudar fenômenos variados, sejam eles matemáticos, do cotidiano, físicos, biológicos, econômicos etc. É importante lembrar que relações funcionais que ocorrem na realidade são raramente descritas com exatidão por fórmulas algébricas, gráficos ou tabelas. No entanto, essas relações são, frequentemente, modeladas por expressões algébricas, gráficos e tabelas. Esses, como modelos, são aproximações das situações em estudo. Tabelas numéricas que as aproximam em configuração finita são frequentemente modelos convenientes para estudá-las. Representações gráficas também são criadas para estudar diversas situações funcionais da realidade; dentre os exemplos mais conhecidos podemos citar os eletrocardiogramas, eletroencefalogramas e sismogramas.

Uma das concepções de função presentes entre alunos manifesta-se ao se referirem ou admitirem o uso da palavra função quase que somente quando esse objeto encontra-se representado por uma expressão algébrica. Vários exemplos de funções que não podem ser descritas por fórmulas estão implicitamente presentes nas discussões apoiadas em gráficos estatísticos oferecidas por diversos livros didáticos de ensino médio. Frequentemente, os textos didáticos não se referem explicitamente à função subjacente aos gráficos estatísticos, apesar de poderem estar a um passo da introdução do conceito de função ou mesmo no capítulo dedicado a funções. Além disso, o aluno precisa ser alertado para o fato de que um gráfico estatístico de uma função é diferente de um gráfico cartesiano da mesma função, até por causa das próprias regras de construção de cada tipo de gráfico.

O estudo de gráficos estatísticos (gráfico de linhas ou curvas, em barras, em setores, pictogramas...) é muito interessante pela importância do desenvolvimento da leitura e da interpretação desses recursos de representação de dados numéricos. A compreensão das informações assim codificadas nos meios de comunicação variados hoje em dia pode ser vista como uma questão de cidadania.

Consideramos que a Estatística é uma área de aplicação rica em exemplos interessantes de situações funcionais e que o estudo de dados da realidade pode motivar os alunos e ajudá-los no desenvolvimento da compreensão do que é uma função e como funções se comportam, no que concordamos com [14].

Pode ser muito proveitoso para o aluno identificar as funções subjacentes a esses gráficos de linha, barras etc., o domínio da variável independente e esboçar o gráfico cartesiano da mesma, quando possível. Além do aluno deparar-se com funções dadas por uma descrição verbal, sem expressão algébrica, seria alertado para as diferenças entre gráficos estatísticos e cartesianos. Uma característica dos gráficos estatísticos pode ser logo notada: as unidades de medida empregadas nos dois eixos podem ser bem diferentes, o que raramente acontece com gráficos cartesianos nos livros didáticos. Esta é uma característica interessante e que pode preparar o aluno para o estudo de gráficos de funções construídos com tecnologias digitais. Nesses ambientes a geração de gráficos com escalas diferentes nos dois eixos é frequente, e pode ser fundamental, pois a escolha das escalas adequadas à resolução de um problema depende do próprio, [10].

É importante observar que as regras de produção dos diferentes tipos de gráficos estatísticos são diferentes entre si e distintas das regras para construção de gráficos cartesianos ortogonais. Essas diferenças também precisam ser explicitadas, pois a utilização do termo "gráfico" pode ficar bastante vaga para o aluno, até mesmo pela rica polissemia dessa palavra. Esse termo pode ser usado com sentidos amplos e bem distintos, além do significado presente em representações gráficas de funções ou de dados estatísticos. "Gráfico" pode denotar um esquema, desenho, esboço ou croquis, referir-se a grafia, artes gráficas, equipamentos gráficos 
etc. A dificuldade dos alunos com a construção, interpretação e utilização de gráficos de funções é bastante mencionada na literatura. A polissemia do termo "gráfico" pode acrescentar um complicador a essa área.

A seguir, exemplificamos resumidamente o que sugerimos acima, considerando dois exemplos de gráficos estatísticos.

Exemplo 1. Vamos identificar as funções subjacentes ao gráfico estatístico na Figura 1. Há cinco funções subtendidas no gráfico estatístico (de linhas) apresentado na Figura 1. Todas elas estão definidas em um conjunto de valores temporais (trimestres de 2007.I a 2012.II), e associam a cada um dos números desse conjunto (que graduam o eixo horizontal - representativos de trimestres) a taxa de variação trimestral do $\mathrm{PIB}^{2}$ de um dentre os cinco países listados na base do gráfico. O procedimento de ligar os pontos de um gráfico de pontos isolados por uma curva contínua é empregado muitas vezes como um recurso para auxiliar a visualização do comportamento da variável estatística.

Essas funções não têm representação algébrica e suas correspondentes representações em gráficos cartesianos são constituídas por pontos isolados. Esses gráficos estatísticos são construídos a partir de tabelas geradas por processos quantitativos variados, incluindo resultados de pesquisas realizadas junto às empresas e às famílias; essas tabelas não são obtidas calculando-se valores de funções expressas algebricamente. É importante observar que tais funções são de fato aproximações, restritas a certo intervalo temporal, da função que associa, a valores temporais específicos, determinadas informações numéricas sobre o PIB.

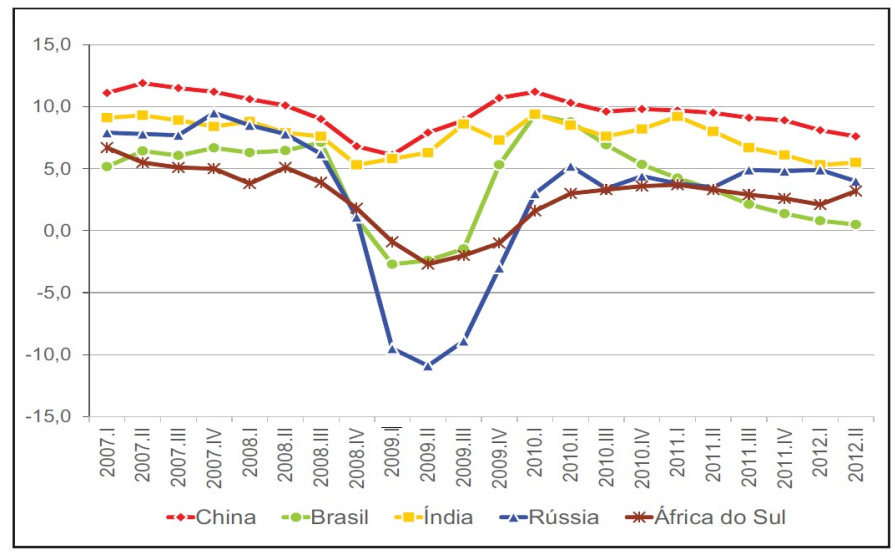

Figura 1: Série da taxa de crescimento trimestral ${ }^{4}$ do PIB dos BRICS até o $2^{\circ}$ trimestre de 2012 - Elaborado pela Coordenação de Contas Nacionais - IBGE.

Exemplo 2. Consideremos os dados apresentados na Figura 2 e o gráfico estatístico (de setores) da Figura 3, que foi construído a partir da tabela da Figura 2.

A tabela da Figura 2, construída com dados de pesquisa domiciliar, representa a função $g$ que associa, a cada intervalo de valores de rendimento em salários mínimos, o percentual de pessoas com rendimento no intervalo $^{5}$. Essa função pode ser vista como uma função $g$ definida no conjunto de intervalos de $\mathbb{R}$ que

\footnotetext{
${ }^{2}$ Total de bens e serviços produzidos no país, descontadas as despesas com os insumos utilizados no processo de produção durante o período.

${ }^{4}$ Refere-se à taxa de variação do PIB no trimestre, em relação ao mesmo trimestre do ano anterior.

${ }^{5}$ Os dados da tabela apresentam alguma margem de erro, assim, de fato, $g$ é uma aproximação da função que associa, a intervalos de valores de rendimento em salários mínimos, o percentual de pessoas com esses rendimentos dentre as entrevistadas.
} 
Pessoas de 10 anos ou mais de idade, ocupadas na semana de referência por classes de rendimento nominal mensal de todos os trabalhos Rio de Janeiro - 2000

\begin{tabular}{lcr}
\hline Classes de Rendimento em SM ${ }^{(1)}$ & Total de Pessoas & $\%$ \\
\hline Até 1 & 807.292 & 14,6 \\
Mais de 1 a 2 & 1.403 .205 & 25,4 \\
Mais de 2 a 3 & 786.826 & 14,2 \\
Mais de 3 a 5 & 899.220 & 16,3 \\
Mais de 5 a 10 & 847.932 & 15,3 \\
Mais de 10 a 20 & 344.141 & 6,2 \\
Mais de 20 & 206.519 & 3,7 \\
Sem Rendimento & 131.014 & 2,4 \\
Total & 5.527 .270 & 100,0 \\
\hline Fonte: IBGE, Censo Demográfico 2000. Tabulação Avançada. & \\
(1) Salário mínimo utilizado pelo IBGE: R\$ $151,00$. &
\end{tabular}

Figura 2: Tabela do Exemplo 2

definem as classes de rendimento consideradas. Tais intervalos e a expressão algébrica da função $g$ estão descritos abaixo:

$$
\begin{array}{r}
I_{0}=\{0\}, I_{1}=(0,1], I_{2}=(1,2], I_{3}=(2,3], I_{4}=(3,5], I_{5}=(5,10], I_{6}=(10,20], I_{7}=(20, \infty), \\
g\left(I_{0}\right)=2,4 ; g\left(I_{1}\right)=14,6 ; g\left(I_{2}\right)=25,4 ; g\left(I_{3}\right)=14,2 ; g\left(I_{4}\right)=16,3 ; g\left(I_{5}\right)=15,3 ; g\left(I_{6}\right)=6,2 ; g\left(I_{7}\right)=3,7
\end{array}
$$

Não faz sentido falar de gráfico cartesiano dessa função. Por outro lado, tanto a tabela da Figura 2 como o gráfico de setores da Figura 3 são muito mais informativos do que a "fórmula" a ela associada.

Os livros didáticos vêm apresentando vários gráficos estatísticos, muitos outros estão presentes em jornais e revistas; alguns podem ser criados com sugestões das atividades no site do IBGE Vamos Contar ${ }^{6}$ Não faltam oportunidades para os alunos defrontarem-se com funções que não podem ser expressas por fórmulas algébricas.

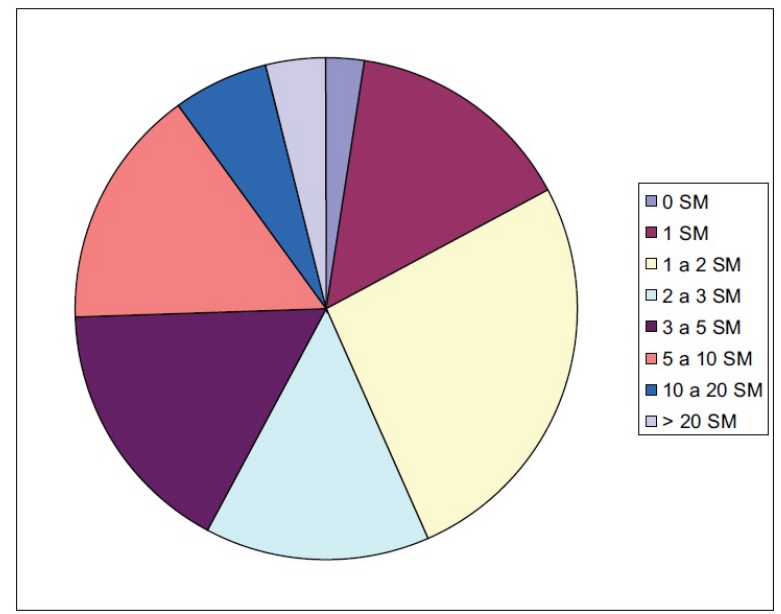

Figura 3: Gráfico de setores do Exemplo 2

\footnotetext{
${ }^{6}<$ http://vamoscontar.ibge.gov.br>.
} 


\section{Um problema de otimização}

O problema aqui apresentado faz parte de uma série de atividades que desenhamos, todas elas estruturadas em torno da resolução de problemas de otimização dados por descrição verbal, visando propiciar um avanço na construção da concepção de função por parte dos alunos. Nosso objetivo é que o aluno, além de calcular valores específicos de uma função dada por uma fórmula, ou manipular sua expressão, possa evoluir para uma concepção segundo a qual ele perceba uma função como recebendo valores e retornando valores, um ato imaginado, sem necessidade de efetuar cálculos, raciocinando sem o apoio de uma fórmula. Além disso, as atividades têm por objetivo: avançar na problemática da leitura e interpretação de enunciados pelos alunos, na discriminação entre constantes e variáveis, no uso de representações múltiplas de funções (por esquemas gráficos, tabela, representação algébrica, representação gráfica), nos processos de justificativa de resolução de problema e no desenvolvimento do conceito de aproximação de um número real. Várias dessas atividades estão associadas a um programa de visualização digital ${ }^{7}$ que permite examinar dinamicamente a relação entre a situação em estudo e um modelo matemático que a representa, além de permitir cálculos e verificação de algumas respostas às questões propostas ao longo das mesmas.

A seguir mostramos o enunciado e o encaminhamento de uma das atividades desse conjunto de tarefas, acompanhados de algumas observações sobre os objetivos das diferentes etapas segundo as quais o trabalho nelas apoiado pode ser implementado com alunos em sala de aula. É importante, em uma sala ou laboratório, que o professor estabeleça um diálogo constante com os alunos e que, literalmente "andando pela sala" comunique-se com os alunos, procurando reconhecer seus sucessos e dificuldades.

\section{UM PROBLEMA DE OTIMIZAÇÃO}

Considere um cilindro circular reto inscrito numa esfera cujo raio mede 3 metros. Seja $r$ a medida, em metros, do raio da base do cilindro.

(a) Dê o domínio da função que fornece o volume $V$ do cilindro, em termos de $r$.

(b) Determine uma aproximação do valor do raio do cilindro de volume máximo dentre os que podem ser inscritos na esfera de raio 3 , com um erro menor do que 0,02 .

\section{Implementação do Item $(\mathbf{a})^{8}$}

Etapa 1: Trabalho inicial orientado para a leitura do enunciado, objetivando algum nível de compreensão do problema. Esclarecimentos sobre o significado de termos técnicos. Alguns alunos podem não lembrar o significado de "um cilindro está inscrito numa esfera".

Etapa 2: Perguntas dirigidas aos alunos e discutidas em conjunto: O que varia e o que permanece constante no contexto do problema estudado? Quantos cilindros circulares retos com raio da base $r=1$ existem? Quantos cilindros circulares retos com raio da base $r=1$ podem ser inscritos numa esfera de raio 3? Quantos cilindros circulares retos com eixo de rotação na direção vertical podem ser inscritos numa esfera de raio 3 ?

Ao longo da discussão, é importante propor aos alunos que esbocem a lápis algumas figuras representativas das situações analisadas. Alguns alunos podem esboçar "figuras no ar" com as mãos, acompanhadas de explicações verbais.

Observação: Sem perda de generalidade, consideram-se, a partir daqui, os cilindros circulares retos com eixo de rotação na direção vertical.

\footnotetext{
${ }^{7}$ Ver em <http://www.uff.br/cdme/pot/> ou <http://www.cdme.im-uff.mat.br/pot/>. Programação: Humberto José Bortolossi. Idealização: Gilda de La Rocque Palis, Silvana Marini Rodrigues Lopes e Humberto José Bortolossi.

${ }^{8} 7$ Daqui em diante, todas as medidas de $r$ estão expressas em metros.
} 
Etapa 3: Solicitações dirigidas aos alunos e discutidas em conjunto: Faça um esboço, a lápis, de alguns cilindros inscritos em uma mesma esfera e procure visualizar qualitativamente a variação da altura dos cilindros inscritos na esfera de raio 3 à medida que o valor do raio cresce. Idem como o volume dos cilindros varia com $r$.

Nessa etapa é proposto aos alunos que verbalizem a percepção qualitativa das variações na altura e no volume do cilindro, enquanto consideram variações no raio do cilindro inscrito na esfera. Esperamos que os alunos venham a concluir que a altura decresce, à medida que $r$ cresce, mas que tenham dificuldades para formular uma conjectura correta sobre o comportamento da função que, a cada valor de $r$, associa o volume do correspondente cilindro.

Etapa 4: Solicitação dirigida aos alunos e discutida em grupo: Considere o cilindro circular reto com raio da base $r=1$ inscrito numa esfera de raio 3. Determine a altura desse cilindro. Determine o volume desse cilindro. Repita com $r=2, r=0, r=20, r=3, r=0,1, r=3,001$. Esperamos que muitos alunos venham a concluir que os números $0,20,3$ e 3,001 não são admissíveis como valores para $r$, sem necessidade de "fazer as contas".

Etapa 5: Pergunta dirigida aos alunos e discutida em conjunto: Quais valores a variável $r$ (medida do raio do cilindro inscrito na esfera de raio 3) pode assumir? Qual o domínio da função que relaciona o valor do volume $V$ do cilindro inscrito na esfera de raio 3 com o valor do raio $r$ da base?

Procuramos aqui verificar se o aluno aceita o domínio da função, no caso o intervalo (0,3), a partir de uma reflexão sobre os possíveis valores de $r$, sem considerar a expressão algébrica da função.

Implementação do Item (b) A partir daqui, a resolução do problema prossegue, agora usando recursos algébricos, gráficos ou de Cálculo Diferencial.

Etapa 6: Pergunta dirigida aos alunos e discutida em grupo: Qual a expressão algébrica da função $h$ que, a cada valor de $r$, associa a altura $h(r)$ do cilindro circular reto de raio $r$ inscrito na esfera de raio 3 ? Qual a expressão da função $V$ que, a cada valor de $r$, associa o volume $V(r)$ do cilindro de raio $r$ inscrito na esfera de raio 3 ?

A variação de $h$ e de $V \operatorname{com} r$ são expressas agora quantitativamente por fórmulas. As respostas esperadas são $h(r)=\sqrt{36-4 r^{2}}$ e $V(r)=\pi r^{2} \sqrt{36-4 r^{2}}$.

Etapa 7: Solicitação dirigida aos alunos: Organize uma resolução justificada para responder a questão, do Item (b).

Nessa etapa, a resolução esperada (cálculo gráfico ou diferencial, com papel e lápis e/ou alguma tecnologia digital) depende do nível de estudos dos alunos.

Um aluno que ainda não estudou Cálculo Diferencial pode fornecer uma resposta à questão utilizando uma representação gráfica da função $V$ cuja representação algébrica ele já conhece (ver Etapa 6). Um gráfico da função $V$, gerado em computador ${ }^{9}$, pode ser visto na Figura 4; nele podemos visualizar um valor de $r$ no qual a função $V$ atinge um máximo absoluto. Designemos por $\alpha$ esse valor de $r$ no qual a função $V$ tem um máximo absoluto.

O que pretendemos é achar uma aproximação de $\alpha$ com um erro menor do que 0,02 . Para isso, podemos gerar um gráfico de $V$ restrito a um intervalo de amplitude menor ou igual a 0,02 e contendo $\alpha$. O gráfico de $V$ restrito ao intervalo $[2,44 ; 2,46]$ que se encontra apresentado na Figura $5^{10}$ tem essas propriedades. Esse gráfico permite responder a questão. Uma resposta para o problema é 2, 45, pois como $\alpha$ e 2,45 pertencem ao intervalo $[2,44 ; 2,45]$ temos que $|\alpha-2,45|<0,02$. É importante observar que existe uma infinidade de respostas à questão do Item (b).

Caso o aluno já tenha cursado Cálculo Diferencial, o valor de $r$ no qual a função $V$ tem um máximo global pode ser obtido pela análise de cálculos algébricos envolvendo a função derivada de $V$. Nesse

\footnotetext{
${ }^{9}$ Diversos recursos digitais estão disponíveis para a geração de gráficos de funções.

${ }^{10}$ Existe uma infinidade de intervalos com as propriedades requeridas.
} 
caso o aluno irá chegar a $\alpha=\sqrt{6}$ e essa é uma resposta à pergunta do enunciado, pois " $\sqrt{6}$ é uma aproximação de $\sqrt{6} " 11$.

Se o aluno resolver a questão dessa forma e desejar verificar, graficamente, se a sua resposta é plausível, ele poderá fazê-lo num gráfico como o da Figura 5, lá visualizando um truncamento do desenvolvimento decimal de $\alpha$; por exemplo, o truncamento de $\sqrt{6}$ na terceira casa decimal, que é 2,449 .

Caso o aluno que já cursou Cálculo Diferencial não consiga efetuar os cálculos algébricos necessários para a resolução anterior, uma resposta do problema também pode ser obtida graficamente utilizando um gráfico da derivada da função $V$.

A primeira tarefa desse tipo realizada em sala com um grupo de alunos que ainda não cursou Cálculo Diferencial tem sido trabalhada com as várias etapas descritas anteriormente. À medida que os alunos vão progredindo, o número de etapas vai sendo reduzido. Mas é importante observar que sempre procuramos que os alunos respondam à pergunta sobre o domínio da função em questão antes de empregar um método gráfico ou analítico para estudá-la.

Os cálculos solicitados na Etapa 4 (inclusive a não aceitação de números fora do domínio) e a visualização dos cilindros gerados para cada valor de $r$ podem ser realizados com recursos do software do Problema do Cilindro Inscrito numa Esfera ${ }^{12}$. No mesmo software, o aluno pode visualizar qualitativamente a variação do volume dos cilindros circulares retos inscritos na esfera de raio 3 à medida que o valor do raio cresce, e além disso, verificar sua resposta às etapas 5 e 6 (determinação do domínio e da expressão da função a otimizar). A visualização da articulação entre o problema geométrico em estudo e o gráfico da função que modela o problema reforça a compreensão da relação funcional estudada. Uma variante dessa atividade consiste em determinar o valor da altura do cilindro de volume máximo dentre os que podem ser inscritos na esfera de raio 3 .

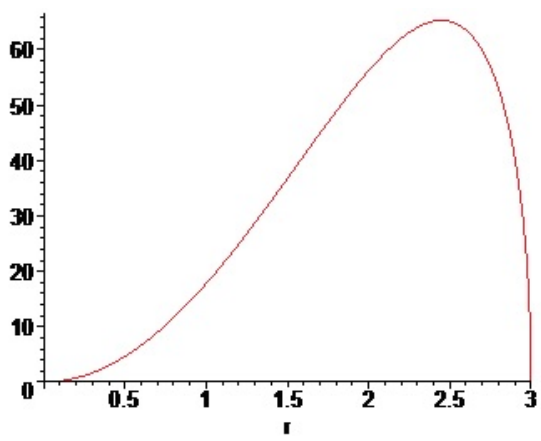

Figura 4: Gráfico de $V$ em função de $r$ no intervalo $(0,3)$.

Os alunos que vêm realizando esse tipo de atividade têm mostrado progressos na compreensão do conceito de domínio de funções dadas por representações diversas. Também verificamos um aumento no percentual de alunos que são bem-sucedidos em problemas de otimização cujos enunciados não trazem a expressão algébrica da função a estudar. Esperamos que as ideias aqui discutidas contribuam para o desenvolvimento de atividades voltadas à melhoria do aprendizado de funções por nossos alunos.

\footnotetext{
${ }^{11}$ É importante salientar que, para muitos alunos, uma aproximação de um número é sempre um número decimal finito.

${ }^{12}$ Esse apoio computacional pode ser encontrado em <http://www.uff.br/cdme/eci/> ou <http://www.cdme. im-uff.mat.br/eci/>.
} 


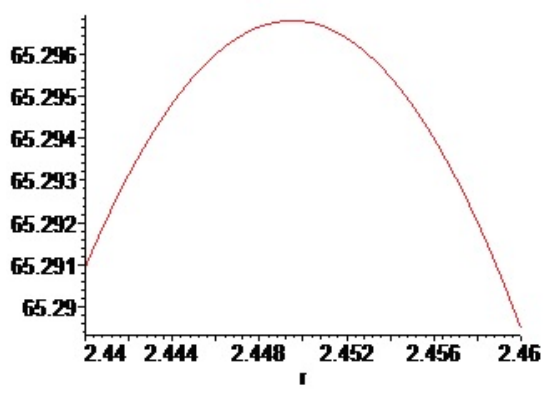

Figura 5: Gráfico de $V$ em função de $r$ no intervalo $[2,44 ; 2,46]$.

Concluindo, lembro que "organizar e dirigir situações de aprendizagem", incluindo "trabalhar a partir das representações dos alunos" e "administrar a progressão das aprendizagens", como planejado ao longo do problema aqui discutido, são algumas das novas competências pedagógicas profissionais para ensinar, segundo [13].

\section{Referências}

[1] Akkoç, H.; Tall, D. A A Mismatch between Curriculum Design and Student Learning: The Case of The function Concept. Hewitt, D.; Noyes, A. (Eds.), Proceedings of The Sixth British Congress of Mathematics Education, University of Warwick, p. 1-8, 2005.

[2] Carlson, M. P. A Cross-Sectional Investigation of The Development of The Function Concept. Schoenfeld, A. H.; J. Kaput, J.; Dubinsky, E. (Eds.), CBMS Issues in Mathematics Education: Research in Collegiate Mathematics Education, American Mathematical Society, n. 7, p. 114$162,1998$.

[3] Chorlay, R. From Historical Analysis to Classroom Work: Function Variation and Long-term Development of Functional Thinking. Proceedings of CERME 6, Lyon, France, 2009.

[4] Duval, R. Semiósis e Pensamento Humano: Registros Semióticos e Aprendizagens Intelectuais. Livraria da Física, São Paulo, 2009. (Obra original publicada em 1995.)

[5] Koedinger, K. R.; Tabachneck, H. J. M. Two Strategies are Better than One: Multiple Strategy Use in Word Problem Solving. Annual Meeting of rhe AERA, New Orleans, LA, 1994.

[6] Morgan, C. What is A Definition for In School Mathematics? European Research in Mathematics Education IV: Proceedings of the Fourth Congress of the European Society for Research in Mathematics Education, Barcelona, p. 861-871, 2006.

[7] Oehrtman, M. C.; Carlson, M. P.; Thompson, P. W. Foundational Reasoning Abilities that Promote Coherence in Students' Understandings of Function. Carlson, M. P.; Rasmussen, C. (Eds.), Making The Connection: Research and Practice in Undergraduate Mathematics, Mathematical Association of America, p. 27-42, 2008.

[8] Palis, G. L. R. Tecnologia, Gráficos e Equações. Revista do Professor de Matemática, Sociedade Brasileira de Matemática, n. 26, p.30-38, 1994.

[9] Palis, G. L. R. Gráficos de Funções em Calculadoras e com Lápis e Papel. Educação e Matemática, Associação de Professores de Matemática de Portugal, n. 45, p. 37-40, 1997. 
[10] Palis, G. L. R.; Ipina, L. Procurando Um Equilíbrio Entre O Que Se Pode "Ver" e O Que Se Pode "Imaginar". Revista Educação Matemática Pesquisa, Educ-SP, v. 1, n. 2, p. 31-46, 1999.

[11] Palis, G. L. R. Uma Análise das Concepções Mentais Subjacentes à Produção e Interpretação de Gráficos de Funções. I Colóquio de História e Tecnologia no Ensino de Matemática (I HTEM), v. 1, p. 251-260, 2002.

[12] Palis, G. L. R. O Conceito de Função: da Concepção Ação à Concepção Processo. Desenvolvimento de tarefas instrucionais. Boletim do LABEM (Laboratório de Educação Matemática) da Faculdade de Educação da UFF, ano 2, n. 2, 2011.

[13] Perrenoud, P. Dez Novas Competências para Ensinar. Artmed, 2000.

[14] Rossman, A. J. Integrating Data Analysis into Precalculus Courses. Hastings, N. B. (Ed.), A Fresh Start for Collegiate Mathematics. Rethinking The Courses below Calculus. MAA Notes, v. 69 , p. 169-177, 2006.

[15] Thompson, P. W. Students, Functions, and The Undergraduate Curriculum. Dubinsky, E.; Schoenfeld, A. H.; Kaput, J. J. (Eds.), CBMS Issues in Mathematics Education: Research in Collegiate Mathematics Education, American Mathematical Society, n. 4, p. 21-44, 1994.

[16] Vinner, S. The Role of Definitions in The Teaching and Learning of Mathematics. Tall, D. (Ed.). Advanced Mathematical Thinking. Kluwer, 1991.

Gilda Palis

PUC-Rio

<gildalrpuc@hotmail.com>

Recebido: 2013

Publicado: 2013 